Abstract ID: 71

Clinical Medicine

Poster

\title{
Knowledge, Practice and Barriers of Pap Smear Screening among Patients in Obstetrics and Gynaecology Ward of the Largest Government Hospital in Pahang
}

Nur Farha Adnin Binti Azmi ${ }^{1}$, Irrah Jefri ${ }^{1}$, Nurul Nabilah Faisal ${ }^{1}$, Kamarul Bahyah Mustaffa ${ }^{2}$, Karimah Hanim bt Abd Aziz ${ }^{3}$, Nabilah Ong ${ }^{2}$

${ }^{1}$ Kulliyyah of Medicine, International Islamic University Malaysia

${ }^{2}$ Department of Obstetric and Gynaecology, Kulliyyah of Medicine, International Islamic University Malaysia

${ }^{3}$ Department of Community Medicine, Kulliyyah of Medicine, International Islamic University Malaysia

Presenter: Nur Farha Adnin Binti Azmi

Introduction: Cervical cancer is the third most common cancer among women in Malaysia. It has the advantage of having the Pap smear as a reliable screening test. Unfortunately, the screening uptake in Malaysia is still very low. This study was performed to determine the knowledge, practice and barriers of Pap smear screening among sexually active patients in Obstetrics and Gynaecology wards in the largest government hospital in the state of Pahang. Materials and Methods: These cross sectional study was conducted in August 2017 using self-administered validated questionnaire covering the socio-demographic factors, knowledge on Pap smear and risk factors of cervical cancer, practice, and barrier of Pap smear screening. Bivariate analyses were used for associations between the variables. Results: The mean score of knowledge of the 156 women recruited was 52.01. Forty nine percents had good knowledge about Pap smear and only $39.7 \%$ ever had Pap smear screening. Majority of the patients were not aware of the risk factors for cervical cancer. The most common barrier to screening was time constraint (45.5\%). There was statistically significant association between parity and practice of Pap smear $(p<0.001)$ Conclusion: Knowledge and practice of Pap smear among the respondents were inadequate. These results indicated the need to increase effort in providing knowledge of Pap smear to the community. This is important for early abnormal cervical changes detection to further reduce the incidence of cervical cancer in Malaysia. 